\title{
Potencial Antioxidante de Bauhinia Kalbreyeri Harms (FABACEAE)
}

\author{
Elizabeth Murillo ${ }^{1}$, Oscar Lombo ${ }^{2}$, Margarita Tique ${ }^{2}$ y Jonh J. Méndez $^{1}$ \\ Universidad del Tolima, Facultad de Ciencias, (1) Departamento de Química, Grupo de investigación \\ en Productos Naturales, (2) Departamento de Biología, Ibagué, Tolima-Colombia \\ (e-mail: emurillo8@hotmail.com; variopinta9@hotmail.com; jmendez@ut.edu.co)
}

\begin{abstract}
Resumen
En este estudio se evaluó la actividad antioxidante de los extractos acuoso y orgánicos de Bauhinia kalbreyeri Harms, reportada por la medicina folclórica Colombiana como antidiabética. Se evaluaron los extractos midiendo su capacidad de captación de radicales libres utilizando el método del 1,1difenil-2-picrilhidracil (DPPH). Los extractos etanólicos y acuosos, alcanzaron una actividad secuestrante del radical DPPH mayor a $90 \%$ en casi todas las concentraciones $(5-120 \mu \mathrm{g} / \mathrm{ml})$; las concentraciones efectivas fueron bajas, confirmando el alto potencial antioxidante. El poder reductor reveló depender del solvente extractor y del uso de hoja o corteza, pero independiente de la concentración; el extracto etanólico de corteza evidenció alto potencial reductor. Los extractos etanólico y acuoso de corteza mostraron la mayor cantidad de fenoles. La planta puede ser considerada una buena fuente de antioxidantes naturales, que podrían contrarrestar el exceso de radicales libres, y así ejercer las propiedades terapéuticas atribuidas, más que actuar como hipoglicemiante.
\end{abstract}

Palabras claves: Bauhinia kalbreyeri, actividad antioxidante, método del DPPH, radicales libres, poder reductor

\section{Antioxidant Potential of Bauhinia Kalbreyeri Harms (FABACEAE)}

\begin{abstract}
Antioxidant activity of aqueous and organics extracts from the leaf and bark of Bauhinia kalbreyeri Harms it is describes, this plant has been determined by the Colombian ethnomedicine as antidiabetic. The extracts were evaluated for their free radical scavenging activity by DPPH method. Ethanol an aqueous extracts showed the higher DPPH radical scavenging activity than $90 \%$ in almost all the concentration tested $(5-120 \mu \mathrm{g} / \mathrm{ml})$, the efficient concentrations were low, supported the high inhibitory potential. The reducing power reveled dependence on the extractor solvent and the use of leaf or bark, but independent from the concentration; ethanolic extract of bark exhibited a high reductive potential. Ethanolic and aqueous extracts of bark showed greatest phenols amount. The plant can be considered as a good source of natural antioxidants, that could resist the excess of free radicals, hence exert the therapeutic properties attributed, more than act as a hypoglycaemic.
\end{abstract}

Keywords: Bauhinia kalbreyeri, antioxidant activity, DPPH method, free radicals, reducing power 


\section{INTRODUCCIÓN}

Los procesos metabólicos normales de todos los organismos que utilizan oxígeno pueden producir especies reactivas del oxígeno (EROS), se estima que cerca del 2 al $5 \%$ del oxígeno total consumido se convierte en EROS $\left(\mathrm{O}_{2}^{-}, \mathrm{OH}^{-}, \mathrm{H}_{2} \mathrm{O}_{2},{ }^{1} \mathrm{O}_{2}\right.$, entre otros). Quizá la fuente endógena más importante generadora de EROS es la cadena respiratoria mitocondrial, aunque también pueden incluirse algunas reacciones del metabolismo de los prostanoides, la autoxidación de las catecolaminas, la actividad de la xantina oxidasa y la activación de fagocitos y células endoteliales (Ríos de Molina, 2003; Ferrari, 1994).

En situación patológica se incrementan sustancialmente estas especies químicas, provocando una alteración orgánica conocida como estrés oxidativo caracterizado por el daño a biomoléculas, viéndose implicadas en la génesis o exacerbación de numerosos procesos en el aparato cardiovascular (aterosclerosis, cardiopatía alcohólica), sistema neurológico (enfermedad de Parkinson, Alzheimer, traumatismos craneales), aparato ocular (catarata, fibroplasia), aparato respiratorio (cáncer de pulmón), riñón (nefrotoxicidad por metales), artritis reumatoidea (Rodríguez et al., 2001; Elejalde, 2001).

Entre los desórdenes metabólicos crónicos de mayor morbilidad y mortalidad está la diabetes, del 5 al $10 \%$ de la población mundial la padece, y se estima que para el año 2010 cerca de 239 millones de personas pueden ser afectadas (Hamdan y Afifi, 2004). La diabetes y el estrés oxidativo parecen relacionarse (Sabu y Kuttan, 2002; Ugochukwu y Babady, 2002), existe evidencia que demuestra que los niveles elevados de glucosa en la sangre conducen a la autoxidación de esta misma, también la glicosilación no enzimática de proteínas y el aumento del metabolismo de la glucosa por la ruta del poliol-sorbitol son contribuyentes del proceso; en cualquier caso la producción de EROS se incrementa, y más aún en presencia de metales como el hierro (Failla y Kiser, 1981). Las EROS contribuyen a la resistencia a la insulina debido a que interfieren con las vías de señalización inducida por esta hormona, evitan así la traslocación del transportador de glucosa GLUT 4 a la membrana plasmática, por otra parte una descarga de EROS es liberada por los neutrófilos, exacerbando los procesos inflamatorios y elevando la concentración de las EROS bajo condiciones patológicas persistentes (Miceli et al., 2005; Fernández et al., 2004).

Si bien los organismos vivos soportan multitudinarios factores endógenos y exógenos de estrés oxidativo, también poseen numerosos sistemas de defensa antioxidantes regulables, enzimáticos (superóxido dismutasa, la catalasa, la GSH-peroxidasa, las quinonas reductasas y hemoxigenasa) y no enzimáticos (Se, $\mathrm{Zn}$, vitaminas $\mathrm{C}$ y $\mathrm{E}$ y carotenoides) que conforman la defensa antioxidante frente a las EROS (Kim et al., 2003), pero que no siempre resultan ser una barrera efectiva.

Una alternativa válida son los vegetales, quienes contienen amplia variedad de compuestos con capacidad de atrapar EROS: vitaminas, carotenoides, compuestos nitrogenados (alcaloides, aminas, betalaínas) e incluso ciertos terpenoides; quizá los metabolitos más reconocidos por su actividad antioxidante son los de naturaleza fenólica: ácidos fenólicos, flavonoides, quinonas, cumarinas, lignanos, estilbenos y taninos (Bandonien y Murkovicub, 2002; Parejo et al., 2000).

Entre los innumerables vegetales de interés medicinal se encuentran las plantas del género Bauhinia, las cuales crecen principalmente en áreas tropicales del planeta (aproximadamente 300 especies), muchas de ellas son utilizadas en la etnofarmacología de Africa, Asia y América Central y del Sur; para el tratamiento de una amplia variedad de enfermedades. Los estudios fitoquímicos han demostrado que glicósidos esteroídicos, triterpenos, lactonas y flavonoides son los principales componentes químicos de las especies de este género (Da Silva y Filho, 2002; Viana et al., 1999).

En un trabajo recientemente realizado en los laboratorios de Fitoquímica de la Universidad del Tolima-Colombia, se intentó dar soporte científico a la actividad hipoglicemiante atribuída a $B$. kalbreyeri, por las etnias tolimenses, a través de un ensayo in-vivo con ratones aloxanizados. Los resultados revelaron que la planta no muestra reducción de los niveles de glucosa sanguínea a las concentraciones probadas (250-1000 ppm). Sin embargo, las investigaciones han demostrado que muchos vegetales utilizados como antidiabéticos no provocan disminución de las concentraciones de 
glucosa en forma directa, sino que la acción antidiabética la ejercen por medio de su capacidad secuestrante de radicales libres (Luo et al., 2004; Mcune y Johns, 2002).

En este estudio se evaluó la actividad antioxidante de los extractos acuoso y orgánicos de Bauhinia kalbreyeri Harms, a través de la determinación de su capacidad de captación de radicales libres, de su poder reductor y del contenido fenólico total; buscando con ello correlacionar la composición química del vegetal con la habilidad para disminuir las EROS, acreditar el uso etnomédico del vegetal y a su vez hacer una contribución al conocimiento científico de la flora del Tolima-Colombia.

\section{MATERIALES Y MÉTODOS}

\section{Reactivos Químicos}

Se utilizaron: 1,1-difenil-2-picrilhidracil (DPPH), ácido ascórbico (AA), cloruro férrico y ferricianuro de potasio; todos ellos y el resto de los reactivos utilizados en este trabajo, incluídos los solventes, fueron de calidad pura para análisis.

\section{Preparación de los Extractos}

La planta B. kalbreyeri, crece en regiones Colombianas de clima cálido. Hoja y corteza de una planta en óptimo estado de desarrollo vegetativo y fitosanitario, fueron colectadas durante el mes de Abril en la zona suburbana de Ibagué (1350 m.s.n.m., $27 \pm 1^{\circ} \mathrm{C}$ ). La especie fue identificada en el Herbario Nacional de la Universidad Nacional de Colombia y referenciado con el número COL: 509144. El material vegetal se secó a la sombra, se cortó en pequeños trozos, y $10 \mathrm{~g}$ de este fueron dejados en contacto con $200 \mathrm{ml}$ de los diferentes solventes: éter de petróleo, acetato de etilo y etanol, durante $48 \mathrm{~h}$. Los extractos acuosos se prepararon por decocción, en el intento por dar validez científica a la metodología aplicada por las etnias del Tolima-Colombia. El proceso de extracción se repitió hasta el agotamiento; posteriormente se filtró (papel de filtro Whatman No 1), se concentró a través de un evaporador rotatorio $\left(65^{\circ} \mathrm{C}\right)$ para obtener el extracto seco, este fue almacenado a $0{ }^{\circ} \mathrm{C}$ y finalmente a partir de él se dispusieron todas las muestras para la determinación de la actividad antioxidante.

\section{Actividad Antirradical. Método del DPPH}

La actividad captadora de radicales libres de $B$. kalbreyeri, se midió aplicando el método descrito por Ohinishi et al. (1994), con algunas modificaciones. Se preparó una solución $0.1 \mathrm{mM}$ de DPPH en metanol, $1 \mathrm{ml}$ de esta solución se adicionó a $3 \mathrm{ml}$ del extracto vegetal resuspendido en metanol y preparado a diferentes concentraciones $(5-120 \mu \mathrm{g} / \mathrm{ml})$. Después de $30 \mathrm{~min}$ de incubación se leyó la absorbancia a $517 \mathrm{~nm}$. Valores muy bajos de absorbancia de la mezcla reaccionante es indicativo de una alta capacidad secuestrante de radicales libre (CSRL), expresada numéricamente mediante la ecuación (1):

$\%$ CSRL $=[$ Abs DPPH - Abs muestra/Abs DPPH $] \times 100$

Todos los ensayos se realizaron por triplicado y se promediaron las lecturas. Con estos datos se determinó la $\mathrm{CE}_{50}$ (concentración eficiente para obtener el $50 \%$ de la capacidad máxima para captar radicales libres). El ácido ascórbico (AA) fue tomado como referencia.

\section{Poder Reductor}

El poder reductor del vegetal en estudio se determinó por el método de Oyaizu (1986). Brevemente, $1 \mathrm{ml}$ del extracto original $(40-80 \mu \mathrm{g} / \mathrm{ml})$ se mezcló con tampón de fosfato $(5 \mathrm{ml}, 0.2 \mathrm{M}, \mathrm{pH} 6.6)$ y con ferricianuro de potasio $(5 \mathrm{ml}, 1 \%)$. Después de un período de incubación de $20 \mathrm{~min}\left(50^{\circ} \mathrm{C}\right)$, a la mezcla reaccionante se le adicionó $5 \mathrm{ml}$ de ácido tricloroacético $(10 \%)$ y se centrifugó a 3000 r.p.m. (10 $\mathrm{min})$. El sobrenadante de la solución $(5 \mathrm{ml})$ se mezcló con agua destilada $(5 \mathrm{ml})$ y cloruro férrico $(1 \mathrm{ml}, 1 \%)$. La absorbancia se midió a $700 \mathrm{~nm}$, el incremento de esta variable da a entender un aumento en el poder reductor. Los datos mostrados corresponden al promedio de tres determinaciones. 
Se utilizó ácido ascórbico $(5,10,15 \mu \mathrm{g} / \mathrm{ml})$ como referencia.

\section{Contenido Fenólico Total}

La cantidad total de compuestos fenólicos en los extractos se determinó con el reactivo de FolinCiocalteu (FC), de acuerdo al procedimiento descrito por Singleton y Rossi (1965). Un mililitro del extracto preparado a $40 \mu \mathrm{g} / \mathrm{ml}$ se aforó con agua a $50 \mathrm{ml} ; 1 \mathrm{ml}$ de esta solución diluida se mezcló con $2.5 \mathrm{ml}$ del reactivo FC (diluido $1: 1$ con agua destilada) y con $2 \mathrm{ml}$ de carbonato de sodio (7.5\%); la mezcla se calentó $\left(10 \mathrm{~min}, 50^{\circ} \mathrm{C}\right)$, se llevó a temperatura ambiente y se leyó la absorbancia a 760 $\mathrm{nm}$ contrastando contra un blanco. La curva de calibración se preparó con ácido gálico (1.0-50 $\mu \mathrm{g} / \mathrm{ml})$. El contenido fenólico de los extractos se expresó como miligramos equivalentes de ácido gálico por gramo de muestra (mg EAG/g).

\section{Análisis Estadístico}

Los datos son expresados como medias de las pruebas hechas por triplicado. Los valores se sometieron a análisis de varianza de una sola vía mediante SAS System program. Diferencias significativas entre medias fueron calculadas con la prueba de Tukey. Valores de $P \leq 0.05$ se tomaron como significativos.

\section{RESULTADOS Y DISCUSIÓN}

\section{Actividades antioxidantes}

En un ensayo preliminar se determinó la actividad antioxidante de los extractos orgánicos y acuosos de $B$. kalbreyeri, probándolos a un mismo nivel de concentración $(40 \mu \mathrm{g} / \mathrm{mL})$. Los resultados se expresaron como "Capacidad Secuestrante de Radicales Libres" (CSRL), expresada en porcentaje. Todos los extractos mostraron una CSRL superior al $75 \%$, evidenciando un alto potencial antioxidante del vegetal; no obstante, fueron los extractos etanólicos y acuosos de hoja y corteza (EC, EH, AC, AH) los de mayor potencial inhibitorio. Con ellos se continuó el trabajo preparando nuevas concentraciones en un rango de 5 a $120 \mu \mathrm{g} / \mathrm{mL}$, los resultados aparecen en la Tabla 1; adicionalmente se muestra la $\mathrm{CE}_{50}$ (concentración efectiva del extracto que logra atrapar el $50 \%$ de los radicales libres DPPH de una solución preparada a $0.1 \mathrm{mM}$ ).

Tabla 1: Capacidad Secuestrante de Radicales Libres (CSRL), a diferentes concentraciones de los cuatro extractos más activos de $B$. kalbreyeri y concentración efectiva $\left(\mathrm{CE}_{50}\right)$.

\begin{tabular}{|c|c|c|c|c|c|c|c|c|c|}
\hline \multirow{2}{*}{ 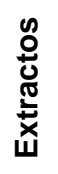 } & \multicolumn{8}{|c|}{ Concentraciones $(\mu \mathrm{g} / \mathrm{mL})$} & \multirow{2}{*}{$\begin{array}{l}\mathrm{CE}_{50} \\
(\mathrm{ppm})\end{array}$} \\
\hline & 5 & 10 & 20 & 40 & 60 & 80 & 100 & 120 & \\
\hline $\begin{array}{l}E \\
C\end{array}$ & $95,42 \pm 0.5$ & $95,57 \pm 0.1$ & $96,01 \pm 0.3$ & $97,70 \pm 0.2$ & $93,61 \pm 0.2$ & $93,28 \pm 0.6$ & $93,79 \pm 0.1$ & $93,84 \pm 0.2$ & 18,75 \\
\hline $\begin{array}{l}\mathrm{E} \\
\mathrm{H} \\
\end{array}$ & $77,05 \pm 1.2$ & $89,06 \pm 0.8$ & $91,00 \pm 1.1$ & $91,40 \pm 0.9$ & $90,88 \pm 0.6$ & $88,54 \pm 0.6$ & $89,32 \pm 0.4$ & $96,06 \pm 1,8$ & 18,42 \\
\hline $\begin{array}{l}\text { A } \\
C\end{array}$ & $79,42 \pm 0.2$ & $83,38 \pm 0.6$ & $89,80 \pm 0.1$ & $96,25 \pm 0.5$ & $96,52 \pm 0.2$ & $95,61 \pm 0.4$ & $95,98 \pm 0.3$ & $95,96 \pm 0.6$ & 27,00 \\
\hline $\begin{array}{l}\mathrm{A} \\
\mathrm{H}\end{array}$ & $80,61 \pm 0.6$ & $84,63 \pm 0.1$ & $92,38 \pm 1.2$ & $96,07 \pm 1.1$ & $96,15 \pm 0.8$ & $95,07 \pm 0.4$ & $95,50 \pm 0.8$ & $94,85 \pm 0.1$ & 28,30 \\
\hline $\begin{array}{l}\text { A } \\
\text { A }\end{array}$ & $78,56 \pm 0.4$ & $79,67 \pm 0.2$ & $95,33 \pm 0.1$ & $96,29 \pm 0.2$ & & & & & \\
\hline
\end{tabular}


Se observa que el vegetal evidencia un alto potencial antioxidante igual o superior al $90 \%$ a concentraciones relativamente pequeñas, efecto que resulta comparable al del ácido ascórbico. El análisis de varianza aplicado dejó ver que existen diferencias significativas entre los extractos, así como también entre las concentraciones probadas $(p \leq 0.05)$. Los valores de la $\mathrm{CE}_{50}$ obtenidos con cada extracto parecen ratificar el potencial de $B$. kalbreyeri para inhibir la propagación de radicales libres. La corteza resultó más activa que la hoja, y el etanol se reveló como el mejor solvente extractor de los compuestos activos de la planta. Otras especies de Bauhinia han sido valoradas en este mismo sentido, mostrando valores entre $5,50 \mu \mathrm{g} / \mathrm{mL}$ y $200 \mu \mathrm{g} / \mathrm{mL}$ (Menezes et al, 2004; Silva et al., 2005), sobre esta base puede pensarse en $B$. kalbreyeri como un vegetal con gran potencial en la prevención de daños inducidos por el desbalance a nivel orgánico de las EROS.

La actividad antioxidante de la planta se complementó midiendo su capacidad para reducir el $\mathrm{Fe}^{+3}$ a $\mathrm{Fe}^{+2}$, monitoreando la formación de un complejo coloreado (reacción tipo Fenton). La figura 1 ilustra el poder reductor de los extractos acuosos y orgánicos probados entre 40 y 80 ppm, comparados con el ácido ascórbico (patrón, 5-15 ppm). Muchos autores sostienen que la actividad antioxidante de algunos extractos polares es debida, al menos en parte, a la presencia de sustancias con grupos hidroxilos, los cuales ejercen su acción por donación de protones (capacidad secuestrante de radicales libre), o bien por interacción, adición o combinación de radicales o por reacciones redox (transferencia de electrones). En cualquier caso es importante la estructura planar y espacial del compuesto antioxidante presente en el extracto (Jung et al., 2006).

Al correlacionar la actividad antioxidante como una función de la naturaleza del solvente extractor, se observaron diferencias significativas entre los extractos $(P \leq 0.05)$. No obstante a que no se encontró diferencias entre los extractos de corteza $(P=0.1520)$, si la hubo para los mismos obtenidos a partir de la hoja $(P<0.05)$. Al aplicar la prueba de Tukey $(P \leq 0.05)$ se notó que en la hoja no hay diferencias significativas entre el extracto acuoso y el etanólico, como tampoco entre el de acetato de etilo y el de éter de petróleo; sin embargo cuando la corteza es la matriz original, la polaridad del solvente juega papel relevante en el potencial reductor (ver Tabla 2; los valores con letras en supraíndice indican que son significativamente diferentes $P \leq 0.05$.).

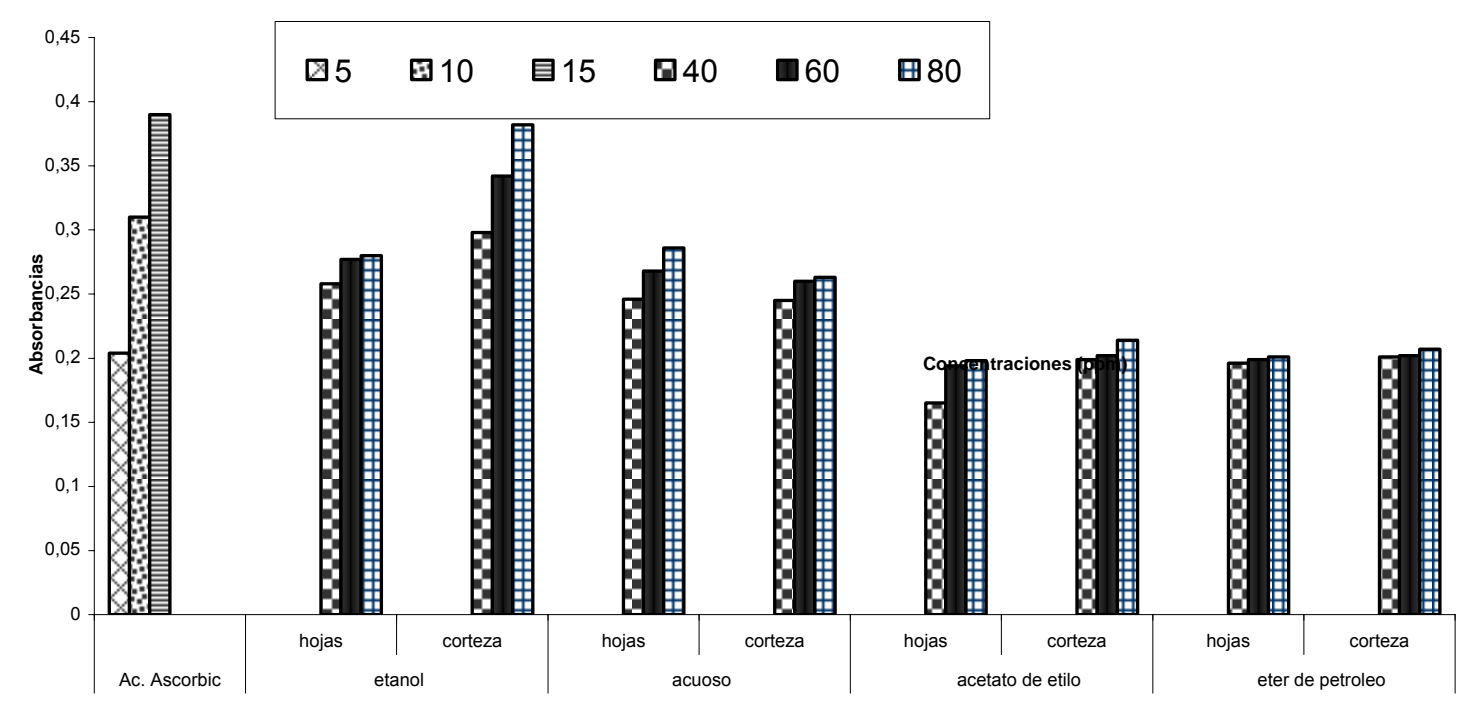

Fig. 1: Poder reductor de los extractos acuoso y orgánicos de B. kalbreyeri comparado con ácido ascórbico. 
El análisis sugiere que la actividad del extracto vegetal, elaborado con acetato de etilo, podría sustituir la del mismo obtenido con agua o éter de petróleo, debido tal vez a su polaridad intermedia entre los otros dos solventes.

El potencial reductor de las especies de Bauhinia ha despertado el interés de muy pocos investigadores, en mayor grado los estudios han determinado la actividad antioxidante de estas plantas midiendo su capacidad captadora de radicales libres (Menezes et al., 2004; Silva et al., 2005; Argolo et al., 2004). Podría decirse que es este uno de los pocos trabajos que a través de diferentes criterios mide la capacidad de una especie de Bauhinia para inhibir la acción de agentes oxidantes.

Tabla 2: Valores promedio $(n=3) \pm D$.E. de las absorbancias obtenidas en la determinación del poder eductor de $B$. kalbreyeri.

\begin{tabular}{|l|l|l|}
\hline Extractos & Hojas & Corteza \\
\hline Etanol & $0,271 \pm 0,011^{\mathrm{a}}$ & $0,340 \pm 0,042^{\mathrm{a}}$ \\
\hline Acuoso & $0,266 \pm 0,02^{\mathrm{a}}$ & $0,256 \pm 0,009^{\mathrm{b}}$ \\
\hline Acetato de etilo & $0,198 \pm 0,018^{\mathrm{b}}$ & $0,205 \pm 0,007^{\mathrm{bc}}$ \\
\hline Éter de petróleo & $0,185 \pm 0,002^{\mathrm{b}}$ & $0,203 \pm 0,003^{\mathrm{c}}$ \\
\hline
\end{tabular}

\section{Contenido Fenólico Total}

La cantidad total de fitofenoles varió en los diferentes extractos entre 0.24 y $26.02 \mathrm{mg}$ de ácido gálico/g de material seco (tabla 3). Los niveles más altos de fenoles se detectaron en los extractos de corteza, etanólico y acuoso. Tal como se esperaba, la respuesta obtenida en los extractos etéreos (corteza y hoja) resultó poco significativa, por lo que los valores fueron omitidos de la Tabla 3.

Tabla 3: Contenido fenólico total (mg EAG/g de peso seco) de extractos acuoso y orgánicos (hoja y corteza) de $B$. kalbreyeri.

\begin{tabular}{|l|c|}
\hline Extractos & $\begin{array}{c}\text { Contenido de fenoles totales en } \\
\text { (mg EAG)/g }\end{array}$ \\
\hline Etanol Corteza & 26,02 \\
\hline Etanol Hojas & 8,4 \\
\hline Acuoso Corteza & 23,19 \\
\hline Acuoso Hojas & 18,47 \\
\hline Acetato de Etilo Corteza & 2,78 \\
\hline Acetato de Etilo Hojas & 4,07 \\
\hline
\end{tabular}

Se realizó un tamizaje fitoquímico, que permitiera conocer con mayor claridad el tipo de metabolito presente en la especie vegetal, utilizando como modelo el extracto etanólico de hojas, teniendo en cuenta que fue uno de los de mayor potencial antioxidante y porque con él se realizó un bioensayo para determinar la actividad hipoglicemiante en ratones aloxanizados (datos no mostrados). El estudio fitoquímico dejó ver abundante contenido de flavonoides, triterpenos/esteroides, lactonas terpénicas y alcaloides, en mediana proporción se detectaron compuestos tipo quinónico, taninos y cardiotónicos. No se encontró respuesta positiva, bajo las condiciones del ensayo, para saponinas y cumarinas. Estos resultados son coincidentes con los de otros estudios de especies de Bauhinia (Gupta et al., 2005; El-hossary, 2000).

La acción antioxidante de los fitofenoles es altamente reconocida como derivada de su capacidad para secuestrar especies reactivas del oxígeno (Sokmen et al., 2005), en tanto que su habilidad para quelar metales, es considerada como el mecanismo menos influyente en su bioactividad como antioxidantes. Sin embargo, si se tiene en cuenta la alta inestabilidad energética y cinética de las EROS, se puede pensar que la capacidad captadora de radicales libres de los polifenoles es la menos factible, y que más bien estos actúan: 1) bloqueando la formación de aquellas especies por interacción con sus precursores, tales como el anión superóxido y el peróxido de hidrógeno, 
predecesores del radical hidroxilo, considerado como el más potente oxidante entre las EROS; 2) a la acción quelante de metales de transición como el cobre y el hierro, los cuales colaboran en la formación de EROS a través de las reacciones de Fenton-Haber-Weiss (Pardo et al., 2005), lo que reduciría la toxicidad de la célula. La acción sinergística de ambos mecanismos podría explicar los valores relativamente bajos obtenidos en la $C_{50}$ y altos en poder reductor alcanzados con $B$. kalbreyeri.

De otra parte, los compuestos de naturaleza fenólica juegan papel importante en los procesos de oxidación lipídica y se les asocia con la actividad antioxidante (Sokmen et al., 2005; Choi et al., 2002), se les considera además con efectos inhibitorios sobre la mutagénesis y carcinogénesis en humanos cuando son incluídos en la dieta diaria a partir de frutas y vegetales (Cai et al., 2004; Namiki, 1990). Específicamente, ácidos fenólicos y flavonoides son típicamente reconocidos como poseedores de actividad antioxidante (Pyo et al., 2004). Ácidos tales como caféico, ferúlico y vainílico, entre otros, son de amplia distribución en el reino vegetal y universalmente se les ha implicado en el papel antioxidante de frutas, verduras, hortalizas y otros vegetales (Pyo et al., 2004). En general se afirma que los fitofenólicos exhiben actividad antioxidante/prooxidante, dependiendo de factores como el potencial reductor de metales, el comportamiento quelante, el $\mathrm{pH}$, las características de solubilidad, de su conformación estructural, de la cantidad de grupos hidroxilos disponibles en la molécula, etc (Mathew y Abraham, 2006).

\section{CONCLUSIONES}

El presente estudio mostró que $B$. kalbreyeri es captadora de radicales libres y que puede ser considerada como una buena fuente de antioxidantes naturales de uso medicinal.

La bioactividad reportada por la etnobotánica colombiana podría fundamentarse no sólo en los diferentes mecanismos ejercidos por compuestos fenólicos que posee (flavonoides, taninos, quinonas), sino además al efecto sinergístico del conjunto de metabolitos secundarios que pudieron evidenciarse en la planta, y a los cuales también se les reconoce esta actividad (alcaloides, terpenos), más que actuar como hipoglicemiante. Es posible también pensar que los efectos de la planta podrían ser acumulativos en el tiempo, lo que contribuiría a explicar los usos etnofarmacológicos.

Una mayor comprensión de las propiedades antioxidantes de los extractos de $B$. kalbreyeri podría tenerse aplicando una amplia variedad de sistemas modelos y utilizando diferentes índices, teniendo en cuenta que la efectividad de los extractos botánicos antioxidantes es, en gran manera, dependiente de las propiedades físicas y químicas del sistema al cual se les adiciona y que los métodos analíticos in vitro adoptados para secuenciar la actividad antioxidante, podrían no ser suficientes a la hora de explicar comportamientos.

\section{AGRADECIMIENTOS}

El grupo de investigación en productos naturales de la Universidad del Tolima (GIPRONUT) apoyó el trabajo reportado en esta comunicación. El soporte técnico del Departamento de Química de la Universidad del Tolima es altamente apreciado.

\section{REFERENCIAS}

Argolo A.C.C., A.E.G. Sant'Ana, M. Pletsch y L.C.B.B. Coelho; Antioxidant activity of leaf extracts from Bauhinia monandra, Bioresource Technology: 95 (2), 229-233 (2004).

Bandonien D. y M. Murkovicub; The detection of radical scavenging compounds in crude extract of borage (Borago officinalis L.) by using an on-line HPLC- DPPH method, J. Biochem. Biophy: Methods: 53 (1-3), 65-70 (2002).

Cai Y., Q. Luob, M. Sunc y H. Corke; Antioxidant activity and phenolic compounds of 112 traditional Chinese medicinal plants associated with anticancer, Life Sciences: 74 74, 2157-2184 (2004). 
Choi C.W. y otros siete autores; Antioxidant activity and free radical scavenging capacity betweenKorean medicinal plants and flavonoids by assay-guided comparison, Plant Science: 163, 1161-1168 (2002).

Da Silva K.L. y V.C. Filho; Plantas do gênero bauhinia: composição química e potencial farmacológico. Química Nova: 25, 55-61 (2002).

Elejalde J.I.; Estrés oxidativo, enfermedades y tratamientos antioxidantes, An. Med. Interna: 18 (6), 145-152 (2001).

El-hossary M.A.; Study of the flavonoid content of Bassia muricata L. and Bauhinia racemosa Lam, Bullet. Faculty Phar. Cairo University: 38, 93-97 (2000).

Failla, M.L y R.A Kiser; Altered tissue content and cytosol distribution of trace metal in experimental diabetes, Journal of Nutrition, Bethesda.: 111 (11) (sep.), 1900-1909 (1981).

Fernández A.C., A.D. Cromarty y C. Albrecht; The antioxidant potential of Sutherlandia frutescens, Journal of Ethnopharmacology.: 95, 1-5. (2004).

Ferrari R. ; Oxygen free radicals at myocardial level: effects of ischemia and reperfusion, Adv. Exp. Med. Biol.: 366, 99-111 (1994).

Gupta M. y otros seis autores; Anti-inflammatory, analgesic and antipyretic effects of methanol extract from Bauhinia racemosa stem bark in animal models, J. Ethnopharmacol.: 98 (3), 267-273 (2005).

Hamdan I.I. y F.U. Afifi; Studies on the in vitro and in vivo hypoglycemic activities of some medicinal plants used in treatment of diabetes in Jordanian traditional medicine, J. Ethnopharmacol: 93 (1), 117-121 (2004).

Jung C.H. y otros cuatro autores; Antioxidant properties of various solvent extracts from wild ginseng leaves. Food Sci. Tecnol.: 36 (3), 266-274 (2006).

Kim K.S. y otros seis autores; Anti-oxidant activities of the extracts from the herbs of Artemisia apiacea, J. Ethnopharmacol.: 85 (1), 69-72 (2003).

Luo Q., Y. y otros cuatro autores; Antioxidant activity and phenolic compounds of 112 traditional Chinese medicinal plants associated with anticancer. Life Sci.: 74 (17), 2157-2184 (2004).

$\mathrm{M}^{\mathrm{c}}$ cune L.M. y T. Johns; Antioxidant activity in medicinal plants associated with the symptoms of diabetes mellitus used by the Indigenous Peoples of the North American boreal forest, J. Ethnopharmacol: 82 (2-3), 197-205 (2002).

Mathew S. y E. Abraham; In vitro antioxidant activity and scavenging effects of Cinnamomum verum leaf extract assayed by different methodologies, Food and Chemical Toxicology: 44, 198-206 (2006).

Menezes P.R., E.A. Schwarz y C.A.M. Santos; In vitro antioxidant activity of species collected in Paraná, Fitoterapia: 75 (3-4), 398-400 (2004).

Miceli N. y otros cinco autores; Anti-inflammatory activity of extract and fractions from Nepeta sibthorpii Bentham J. Ethnopharmacol: 97(2), 261-266 (2005).

Namiki M.; Antioxidants/antimutagens in food, Crit. Rev.Food Sci. Nutr.: (29),273-300 (1990).

Ohinishi M. y otros seis autores; Inhibitory effects of chlorogenic acids on linoleic acid, peroxidation and haemolysis, Phytochemistry: 36, 579-583 (1994). 
Oyaizu M.; Studies on the products of browning reaction prepared from glucose amine Japan Journal of Nutrition: 44, 307-315 (1986).

Parejo I. y otros cinco autores; Evaluation of scavenging activity assessed by Co (II)/EDTA-induced luminol chemiluminescence and DPPH (2,2-diphenyl-1-picrylhydrazyl) free radical assay, Journal of Pharmacological and Toxicological Methods: 44 (3), 435-438 (2000).

Pardo G. y otros cinco autores; Mangifera indica L. extract (Vimang) inhibits Fe2+-citrate-induced lipoperoxidation in isolated rat liver mitochondria. Pharrmacol. Res.: 51 (5), 427-435 (2005).

Pyo Y.H., T.C. Lee, L. Logendra y R.T. Rosen, Antioxidant activity and phenolic compounds of Swiss chard (Beta vulgaris subspecies cycla) extracts, Food Chem.: 85, (19), 19-26 (2004).

Ríos de Molina M.C.; El estrés oxidativo y el destino celular, Quím. Viva: 2 (1), 73-80 (2003).

Rodríguez J.M., J.R. Menéndez e Y. Trujillo; Radicales libres en la biomedicina y estrés oxidativo. Rev. Cubana Med. Milit.: 30 (1), 15-20 (2001).

Sabu M.C. y R. Kuttan; Anti-diabetic activity of medicinal plants and its relationship with their antioxidant property, J. Ethnopharmacol.: 81(2), 155-160 (2002).

Silva C.G. y otros catorce autores; Evaluation of antioxidant activity of Brazilian plants, Pharmacology Res.: 52 (1), 229-233 (2005).

Singleton V. L. y J.A. Rossi; Colorimetry of total phenols with phospho molybdic phoshphotungstic acid reagents, citado por MATHEW, Sindhu and ABRAHAM, Emilia. In vitro antioxidant activity and scavenging efects of Cinnamomum verum leaf extract assayed by diferent methodologies, Amer. J. Enology Vitic.: 16, 144 (1965).

Sokmen M. y otrso seis autores; In vitro antioxidant activity of polyphenol extracts with antiviral properties from Geranium sanguineum L. Life Sciences: 76 (25), 2981-2993 (2005).

Ugochukwu N.H. y N.E. Babady; Antioxidant effects of Gongronema latifolium in hepatocytes of rat models of non-insulin dependent diabetes mellitus, Fitoterapia: 73 (7-8), 612-618 (2002).

Viana E.P., R.S. Santa-Rosa, S.S.M.S Almeida y L.S. Santos; Anti-inflammatory and Analgesic Activities of the Crude Extractsfrom Stem Bark of Bauhinia Guianensis. Pharmaceutical Biology: 37 (4), 281-284 (1999). 
